\title{
Cumulative live birth rate after up to three consecutive embryo transfer cycles in women with poor ovarian response
}

\author{
Se Jeong Kim ${ }^{1,2}$, Dayong Lee ${ }^{2,3}$, Seul Ki Kim², ${ }^{2,4}$ Byung Chul Jee ${ }^{2,4}$, Seok Hyun Kim² \\ 'Department of Obstetrics and Gynecology, Fertility Center of CHA Gangnam Medical Center, CHA University School of Medicine, Seoul; ${ }^{2}$ Department \\ of Obstetrics and Gynecology, Seoul National University College of Medicine, Seoul; ${ }^{3}$ Department of Obstetrics and Gynecology, Seoul Metropolitan \\ Government-Seoul National University Boramae Medical Center, Seoul; ${ }^{4}$ Department of Obstetrics and Gynecology, Seoul National University Bundang \\ Hospital, Seongnam, Korea
}

Objective: In the present study, we aimed to retrospectively evaluate the cumulative live birth rate (LBR) after up to three consecutive embryo transfer (ET) cycles, either fresh or frozen, in women with expected poor ovarian response (ePOR).

Methods: We selected 115 women who entered the first in vitro fertilization (IVF) cycle between August 2013 and July 2016. The women were divided into an ePOR group (37 women) and a non-ePOR group (78 women). All women in the ePOR group were $\geq 40$ years old or had serum anti-Müllerian hormone levels of less than $1.1 \mathrm{ng} / \mathrm{mL}$ at the time of the first IVF cycle. Live birth outcomes were monitored until December 2017. The cumulative LBR (with both conservative and optimistic estimates) was calculated according to the serial number of ET cycles.

Results: After up to three ET cycles, the overall cumulative LBR was significantly lower in the ePOR group than in the non-ePOR group (conservative estimate, $10.8 \%$ vs. $44.9 \%$, respectively; optimistic estimate, $14.7 \%$ vs. $56.1 \%$, respectively; log-rank test, $p=0.003$ ).

Conclusion: Women with ePOR exhibited a lower cumulative LBR than women in the non-ePOR group, and this information should be provided to ePOR women during counseling before starting IVF.

Keywords: Embryo transfer; Fertilization in vitro; Live birth; Pregnancy rate

\section{Introduction}

For in vitro fertilization (IVF) treatment cycles, poor ovarian response (POR) is usually defined as fulfillment of at least two of the following Bologna criteria: (1) advanced age ( $\geq 40$ years) or the presence of other risk factors for POR, (2) a previous history of POR (indicated by the collection of $\leq 3$ oocytes under a conventional stimulation protocol), and (3) an abnormal ovarian reserve test, as shown by either

Received: September 23, 2019 · Revised: December 21, 2019 · Accepted: January 2, 2020 Corresponding author: Byung Chul Jee

Department of Obstetrics and Gynecology, Seoul National University Bundang Hospital, 82 Gumi-ro 173beon-gil, Bundang-gu, Seongnam 13620, Korea Tel: +82-31-787-7254 Fax:+82-31-787-4054 E-mail: blasto@snubh.org

This is an Open Access article distributed under the terms of the Creative Commons Attribution Non-Commercial License (http://creativecommons.org/licenses/by-nc/4.0/) which permits unrestricted non-commercial use, distribution, and reproduction in any medium, provided the original work is properly cited. an antral follicle count (AFC) $<5-7$ or a serum anti-Müllerian hormone $(\mathrm{AMH})$ level $<0.5-1.1 \mathrm{ng} / \mathrm{mL}[1]$.

Since few oocytes are retrieved from women with POR, oocyte retrieval is less likely to obtain good-quality embryos in these women than in non-POR patients. Thus, in women with POR, the cycle cancellation rate is generally high, and the pregnancy rate per started cycle is generally low [2]. In a previous report, the live birth rate (LBR) per started cycle was found to be $6 \%$ in women with POR [2]. Since women typically receive several rounds of IVF treatment, the assessment of the cumulative LBR could be important for evaluating the long-term prognosis in specific groups. Nonetheless, the cumulative LBR after multiple IVF treatments in women with POR has rarely been reported.

Polyzos et al. [3] evaluated the cumulative LBR for at least 2 years, from either fresh or frozen embryo transfer (ET) cycles, according to 
the number of oocytes retrieved in the first IVF cycle. When 1,2, and 3 oocytes were obtained in the first IVF cycle, cumulative LBR was approximately $8 \%, 13 \%$, and $19 \%$, respectively. However, those researchers did not evaluate the cumulative LBR in a designated POR group. In women with POR, the cumulative LBR after six IVF cycles was shown to be $14.9 \%$ when estimated conservatively and $35.3 \%$ when estimated optimistically [4]. In that study, the optimistically estimated cumulative LBR was higher than the conservatively estimated cumulative $L B R$, indicating a relatively high proportion of patients who were lost to follow-up. In addition, the researchers defined POR based on the Bologna criteria at the time of the first IVF cycle and presented the cumulative LBR according to age group ( $\leq 30$ years, 31-34 years, 35-37 years, 38-40 years, $41-43$ years, and $>43$ years).

Before a woman starts IVF treatment, the Bologna criterion of a previous history of POR cannot be applied to the definition of POR. Consequently, when starting IVF for the first time, women are defined as having POR if they fulfill two conditions: (1) advanced maternal age ( $\geq 40$ years) or other risk factors for POR and (2) an AFC $<5-7$ or serum AMH level $<0.5-1.1 \mathrm{ng} / \mathrm{mL}$. However, young women sometimes exhibit unexpectedly low serum AMH levels, in which case they are generally considered to belong in the POR group during the first IVF cycle. In addition, women $\geq 40$ years old are generally regarded as belonging in the POR group during the first IVF cycle even if they display normal age-matched levels of serum AMH. Therefore, from a practical viewpoint, it is reasonable to consider the POR criteria to be (1) women of advanced age ( $\geq 40$ years) or with other risk factors for POR or (2) an AFC $<5-7$ or serum AMH level $<0.5-1.1 \mathrm{ng} / \mathrm{mL}$.

In this study, we defined patients with expected POR (ePOR) as (1) those $\geq 40$ years old or (2) those with serum AMH level $<1.1 \mathrm{ng} / \mathrm{mL}$ at the time of the first IVF cycle. Under this definition, we evaluated the cumulative LBR after up to three consecutive fresh or frozen ET cycles in women with ePOR.

\section{Methods}

We selected 115 women who underwent the first IVF cycle between August 2013 and July 2016. Live birth outcomes were monitored until December 2017. Women who underwent oocyte donation cycles and accumulation cycles were excluded. The Institutional Review Board of Seoul National University Bundang Hospital approved this study (IRB No. B-1808-487-112). This study approved the patient's waiver of consent due to the retrospective study design.

The ePOR group included 37 women who were $\geq 40$ years old or who had a serum AMH level $<1.1 \mathrm{ng} / \mathrm{mL}$ at the time of the first IVF cycle. The remaining 78 women were assigned to the non-ePOR group. Serum AMH levels were measured in all women using an
AMH assay kit (AMH Gen II A79765; Beckman Coulter, Brea, CA, USA) with revised instructions (IFU-REF A92268D) within 3 months prior to the first IVF cycle. The measurement range was $0.08-22.50 \mathrm{ng} / \mathrm{mL}$, and the intra- and inter-assay coefficients of variation were $5.4 \%$ and $5.6 \%$, respectively.

Ovarian stimulation was performed with recombinant follicle-stimulating hormone (Gonal-f; Merck Serono, Darmstadt, Germany), recombinant follicle-stimulating hormone and recombinant luteinizing hormone (Pergoveris, Merck Serono), or purified human menopausal gonadotropin (Menopur; Ferring Pharmaceuticals, Kiel, Germany). While the majority of ovarian stimulations were conventional, five mild stimulation cycles and one natural cycle were conducted. A gonadotropin-releasing hormone antagonist or a luteal long protocol consisting of a gonadotropin-releasing hormone agonist was used for pituitary suppression. When two or more follicles $\geq 18 \mathrm{~mm}$ in diameter were visible on an ultrasound examination, $250 \mu \mathrm{g}$ of recombinant human chorionic gonadotrophin (hCG; Ovidrel; EMD Serono, Rockland, MA, USA) was provided. In the natural cycle, when a single follicle reached $\geq 19 \mathrm{~mm}$ in diameter, $\mathrm{hCG}$ was administered. Oocyte retrieval was performed 36-38 hours after hCG injection. The ET cycles analyzed in our study included both fresh and frozen cycles. Clinical pregnancy was defined as the visualization of a gestational sac with the detection of a fetal heartbeat on an ultrasound exam. A live birth was defined as pregnancy lasting for more than 28 weeks of gestation and/or the birth of a living child. Delivery of a multifetal pregnancy was considered one live birth.

We used IBM SPSS ver. 19.0 (IBM Corp., Armonk, NY, USA). The cumulative LBR was calculated according to the serial number of ET cycles. Both conservative and optimistic estimation methods were used to deal with patients who dropped out. The conservative method operated on the assumption that the LBR of patients who dropped out would be 0 , since these patients may have a poor prognosis after ET [5]. The optimistic method involved assuming that the LBR of patients who dropped out was similar to that of patients who continued until the next cycle [5]. The cumulative LBR obtained via the Kaplan-Meier method was compared between the two groups using the log-rank test. The Pearson chi-square test, the Fisher exact test, and the Student $t$-test were used for the statistical analysis of various clinical parameters. A $p$-value $<0.05$ was considered to indicate statistical significance.

\section{Results}

The clinical parameters for the ePOR group and the non-ePOR group in the first IVF cycle are shown in Table 1. As expected, women in the ePOR group were older and exhibited a lower mean level of serum $\mathrm{AMH}$ and a lower mean number of oocytes retrieved in the 
first IVF cycle than subjects in the non-ePOR group. The mean number of ET cycles was $1.5 \pm 0.8$ in the ePOR group and $1.7 \pm 0.7$ in the non-ePOR group ( $p=0.306)$. Frozen ET cycles were performed in $3.5 \%$ ( 2 of 57 women) of the ePOR group and in $18.9 \%$ ( 25 of 132 women) of the non-ePOR group.

Cumulative pregnancy outcomes are presented in Table 2. In the ePOR group (37 women), the cumulative LBR after up to three ET cycles was $10.8 \%$ when estimated conservatively and $14.7 \%$ when estimated optimistically. In the non-ePOR group (78 women), the cumulative LBR after up to three ET cycles was $44.9 \%$ when estimated conservatively and $56.1 \%$ when estimated optimistically. A graphical depiction of the cumulative LBR according to serial ET cycle is shown in

Table 1. Clinical parameters in the first IVF cycle in the study population

\begin{tabular}{lccr}
\hline Variable & ePOR group & $\begin{array}{c}\text { Non-ePOR } \\
\text { group }\end{array}$ & $p$-value \\
\hline No. of women & 37 & 78 & \\
Female age $(\mathrm{yr})$ & $40.0 \pm 3.9$ & $34.1 \pm 3.1$ & $<0.001$ \\
Female body mass index $\left(\mathrm{kg} / \mathrm{m}^{2}\right)$ & $22.7 \pm 3.0$ & $22.0 \pm 3.3$ & 0.244 \\
Serum AMH level $(\mathrm{ng} / \mathrm{mL})$ & $1.08 \pm 1.59$ & $4.32 \pm 2.73$ & $<0.001$ \\
Indications of IVF & & & \\
$\quad$ Male & $4(10.8)$ & $11(14.1)$ & 0.771 \\
Tubal & $2(5.4)$ & $3(3.8)$ & 0.656 \\
Ovulatory & 0 & $5(6.4)$ & 0.174 \\
Endometriosis & $2(5.4)$ & $7(9.0)$ & 0.716 \\
Uterine & $1(2.7)$ & $3(3.8)$ & 1.000 \\
Unexplained & $22(59.5)$ & $48(61.5)$ & 0.831 \\
Combined & $6(16.2)$ & $21(26.9)$ & 0.206 \\
No. of oocytes retrieved & $3.8 \pm 2.9$ & $9.7 \pm 6.9$ & $<0.001$ \\
\hline
\end{tabular}

Values are presented as mean \pm standard deviation or number (\%).

IVF, in vitro fertilization; ePOR, expected poor ovarian responder; $\mathrm{AMH}$, antiMüllerian hormone.

Table 2. Cumulative live birth rate according to embryo transfer cycle

\begin{tabular}{|c|c|c|c|c|c|c|c|}
\hline \multirow{2}{*}{$\begin{array}{l}\text { Embryo } \\
\text { transfer cycle }\end{array}$} & \multirow{2}{*}{$\begin{array}{l}\text { No. of } \\
\text { women }\end{array}$} & \multirow{2}{*}{$\begin{array}{l}\text { No. of clinical } \\
\text { pregnancy }\end{array}$} & \multirow{2}{*}{$\begin{array}{l}\text { No. of live } \\
\text { birth }\end{array}$} & \multirow{2}{*}{$\begin{array}{l}\text { Clinical pregnancy } \\
\text { rate per cycle (\%) }\end{array}$} & \multirow{2}{*}{$\begin{array}{l}\text { Live birth rate } \\
\text { per cycle }(\%)\end{array}$} & \multicolumn{2}{|c|}{ Cumulative live birth rate (\%) } \\
\hline & & & & & & $\begin{array}{l}\text { Optimal } \\
\text { estimation }\end{array}$ & $\begin{array}{l}\text { Conservative } \\
\text { estimation }\end{array}$ \\
\hline \multicolumn{8}{|l|}{ Overall } \\
\hline $1 \mathrm{st}$ & 115 & 25 & 22 & 21.7 & 19.1 & 19.1 & 19.1 \\
\hline 2nd & 56 & 17 & 16 & 30.4 & 28.6 & 42.2 & 33.0 \\
\hline $3 r d$ & 18 & 1 & 1 & 5.6 & 5.6 & 46.0 & 33.9 \\
\hline \multicolumn{8}{|l|}{ ePOR group } \\
\hline $1 s t$ & 37 & 4 & 3 & 10.8 & 8.1 & 8.1 & 8.1 \\
\hline 2nd & 14 & 2 & 1 & 14.3 & 7.1 & 14.7 & 10.8 \\
\hline $3 r d$ & 6 & 0 & 0 & 0 & 0 & 14.7 & 10.8 \\
\hline \multicolumn{8}{|c|}{ Non-ePOR group } \\
\hline $1 s t$ & 78 & 21 & 19 & 26.9 & 24.4 & 24.4 & 24.4 \\
\hline 2nd & 42 & 15 & 15 & 35.7 & 35.7 & 51.4 & 43.6 \\
\hline $3 r d$ & 12 & 1 & 1 & 8.3 & 8.3 & 56.1 & 44.9 \\
\hline
\end{tabular}

ePOR, expected poor ovarian responder.

${ }^{\text {a) }} p=0.003$ by the log-rank test.
Figure 1. The cumulative LBR as assessed by the Kaplan-Meier method differed significantly between the ePOR group and the non-ePOR group (log-rank test, $p=0.003$ ).

\section{Discussion}

In this study, we presented the cumulative LBR after up to three consecutive ET cycles in women with $\mathrm{EPOR}$. We defined women with ePOR as those $\geq 40$ years old or with a serum AMH level $<1.1 \mathrm{ng} / \mathrm{mL}$ at the time of the first IVF cycle. In the ePOR group, the conservative

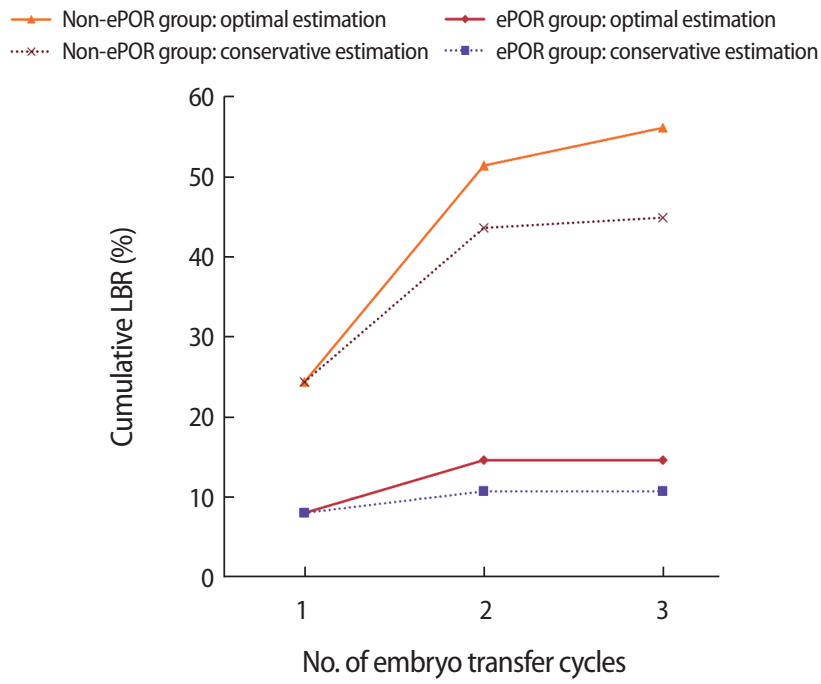

Figure 1. Cumulative live birth rate (LBR) in the expected poor ovarian response (ePOR) and non-ePOR groups according to serial embryo transfer cycle. The cumulative LBR showed a significant difference between the ePOR and non-ePOR groups (log-rank test, $p=0.003$. 
estimate of cumulative LBR after up to three consecutive ET cycles was $10.8 \%$, which is lower than the cumulative LBR after six IVF cycles that was reported by $\mathrm{Xu}$ et al. [4] (14.9\%). However, the cumulative LBR in the present study is very similar to the cumulative LBR reported by Xu et al. [4] for women aged 41-43 years (10.5\%).

Our criteria for ePOR had two components: maternal age and serum AMH levels. The latter is an excellent marker for the prediction of ovarian response or the number of oocytes retrieved after ovarian stimulation [6]. However, it has previously been shown to be a weak predictor for pregnancy and/or live birth in the index cycle [7,8]. Until now, studies analyzing the cumulative pregnancy rate or LBR according to initial serum AMH levels have been lacking. If the cumulative LBR can be assessed using initial serum AMH levels, it follows that initial serum AMH levels could predict the cumulative LBR.

It is quite well known that maternal age is a significant factor in predicting IVF success in the general infertile population. Maternal age has also been reported to be a significant predictor of IVF success in women with POR [9]. Similarly, Xu et al. [4] reported that the cumulative LBR in women with POR diagnosed using the Bologna criteria tended to diminish with increasing age. In a recent study, poor-prognosis women stratified according to the Patient-Oriented Strategies Encompassing IndividualizeD Oocyte Number (POSEIDON) criteria showed a relatively high cumulative LBR in younger age groups, despite the low level of ovarian reserve markers observed in those women [10].

Patients drop out of IVF treatment for a variety of reasons, including economic and psychological reasons [11]. Among these, poor prognosis is an influential factor. When calculating the cumulative pregnancy rate, it is crucial to estimate the number of patients who discontinued treatment. In our study, we used both an optimistic and a conservative estimation method. The actual cumulative LBR is thought to fall between the optimistic and conservative estimates of cumulative LBR. A more accurate cumulative LBR could be obtained if the reasons why patients dropped out were investigated.

Studies of the cumulative LBR in women with POR are scarce. In most published studies, the LBR per cycle was presented as the primary outcome. However, LBR per cycle does not indicate the success rate of following cycles after the failure of previous cycles. From a practical viewpoint, the cumulative LBR for women with POR is very informative because it enables us to understand the long-term prognosis and to determine whether a given woman should continue with subsequent IVF cycles [12]. After three unsuccessful ET cyclestermed repeated implantation failure-IVF treatment is usually terminated or the treatment strategy is modified [13].

In the present study, the cumulative LBR was calculated based on consecutive ET cycles, not consecutive oocyte pickup cycles. Therefore, if transferrable embryos were not obtained after oocyte pickup, those cycles were not counted in the estimation of the cumulative LBR. Women with POR commonly experience cancelled cycles due to inadequate ovarian response, premature follicle rupture, or empty follicle syndrome. As such, substantial numbers of women with POR do not receive ET. In this respect, in women with POR, the cumulative LBR calculated via consecutive oocyte pickup cycles can be assumed to be lower than the cumulative LBR calculated via consecutive ET cycles.

In conclusion, we present the cumulative LBR after up to three consecutive ET cycles in women with ePOR at the time of the first IVF cycle. This finding may be indicative of the reproductive potential of women with ePOR. Because most live births were achieved during the first two cycles, women with POR should be encouraged to pursue at least two cycles of IVF.

\section{Conflict of interest}

Byung Chul Jee has been an editor of Journal of Clinical and Experimental Reproductive Medicine since 2018; however, he was not involved in the peer reviewer selection, evaluation, or decision process of this article. No other potential conflicts of interest relevant to this article were reported.

\section{ORCID}

Se Jeong Kim https://orcid.org/0000-0003-1879-3451

Dayong Lee https://orcid.org/0000-0003-4340-8180

Seul Ki Kim https://orcid.org/0000-0002-1647-6711

Byung Chul Jee https://orcid.org/0000-0003-2289-6090

Seok Hyun Kim https://orcid.org/0000-0003-0649-3224

\section{Author contributions}

Conceptualization: SKK, BCJ, SHK. Data curation: SJK. Formal analysis: SJK, DL. Methodology: SJK, DL, SKK. Project administration: BCJ. Visualization: SJK, BCJ. Writing-original draft: SJK. Writing-review \& editing: all authors.

\section{References}

1. Ferraretti AP, La Marca A, Fauser BC, Tarlatzis B, Nargund G, Gianaroli $L$, et al. ESHRE consensus on the definition of 'poor response' to ovarian stimulation for in vitro fertilization: the Bologna criteria. Hum Reprod 2011;26:1616-24.

2. Busnelli A, Papaleo E, Del Prato D, La Vecchia I, lachini E, Paffoni A, et al. A retrospective evaluation of prognosis and cost-effectiveness of IVF in poor responders according to the Bologna criteria. 
Hum Reprod 2015;30:315-22.

3. Polyzos NP, Drakopoulos P, Parra J, Pellicer A, Santos-Ribeiro S, Tournaye $\mathrm{H}$, et al. Cumulative live birth rates according to the number of oocytes retrieved after the first ovarian stimulation for in vitro fertilization/intracytoplasmic sperm injection: a multicenter multinational analysis including $\sim 15,000$ women. Fertil Steril 2018;110:661-70.

4. Xu B, Chen Y, Geerts D, Yue J, Li Z, Zhu G, et al. Cumulative live birth rates in more than 3,000 patients with poor ovarian response: a 15-year survey of final in vitro fertilization outcome. Fertil Steril 2018;109:1051-9.

5. Raz N, Shalev A, Horowitz E, Weissman A, Mizrachi Y, Ganer Herman $\mathrm{H}$, et al. Cumulative pregnancy and live birth rates through assisted reproduction in women $44-45$ years of age: is there any hope? J Assist Reprod Genet 2018;35:441-7.

6. La Marca A, Sighinolfi G, Radi D, Argento C, Baraldi E, Artenisio $\mathrm{AC}$, et al. Anti-Mullerian hormone (AMH) as a predictive marker in assisted reproductive technology (ART). Hum Reprod Update 2010;16:113-30.

7. Iliodromiti S, Kelsey TW, Wu O, Anderson RA, Nelson SM. The predictive accuracy of anti-Müllerian hormone for live birth after assisted conception: a systematic review and meta-analysis of the literature. Hum Reprod Update 2014;20:560-70.

8. Tal R, Tal O, Seifer BJ, Seifer DB. Antimullerian hormone as predictor of implantation and clinical pregnancy after assisted conception: a systematic review and meta-analysis. Fertil Steril 2015; 103:119-30.

9. Oudendijk JF, Yarde F, Eijkemans MJ, Broekmans FJ, Broer SL. The poor responder in IVF: is the prognosis always poor? A systematic review. Hum Reprod Update 2012;18:1-11.

10. Leijdekkers JA, Eijkemans MJ, van Tilborg TC, Oudshoorn SC, van Golde RJ, Hoek A, et al. Cumulative live birth rates in low-prognosis women. Hum Reprod 2019;34:1030-41.

11. Gameiro S, Boivin J, Peronace L, Verhaak CM. Why do patients discontinue fertility treatment? A systematic review of reasons and predictors of discontinuation in fertility treatment. Hum Reprod Update 2012;18:652-69.

12. Abuzeid MI, Bolonduro O, La Chance J, Abozaid T, Urich M, Ullah $\mathrm{K}$, et al. Cumulative live birth rate and assisted reproduction: impact of female age and transfer day. Facts Views Vis Obgyn 2014; 6:145-9.

13. Margalioth EJ, Ben-Chetrit A, Gal M, Eldar-Geva T. Investigation and treatment of repeated implantation failure following IVF-ET. Hum Reprod 2006;21:3036-43. 